\title{
Coarse-grained collisionless dynamics with long-range interactions
}

\author{
Guido Giachetti, ${ }^{1,2, *}$ Alessandro Santini, ${ }^{3, \dagger}$ and Lapo Casetti $\oplus^{3,4, \$}$ \\ ${ }^{1}$ SISSA, via Bonomea 265, I-34136 Trieste, Italy \\ ${ }^{2}$ INFN, Sezione di Trieste, via Valerio 2, I-34127 Trieste, Italy \\ ${ }^{3}$ Dipartimento di Fisica e Astronomia, Università di Firenze, via G. Sansone 1, I-50019 Sesto Fiorentino, Italy \\ ${ }^{4}$ INFN, Sezione di Firenze, via G. Sansone 1, I-50019 Sesto Fiorentino, Italy \\ and INAF-Osservatorio Astrofisico di Arcetri, Largo E. Fermi 5, I-50125 Firenze, Italy
}

(Received 2 October 2019; accepted 26 May 2020; published 22 June 2020)

\begin{abstract}
We present an effective evolution equation for a coarse-grained distribution function of a long-rangeinteracting system preserving the symplectic structure of the noncollisional Boltzmann, or Vlasov, equation. First, we derive a general form of such an equation based on symmetry considerations only. Then we explicitly derive the equation for one-dimensional systems, finding that it has the form predicted on general grounds. Finally, we use this equation to predict the dependence of the damping times on the coarse-graining scale and numerically check it for some one-dimensional models, including the Hamiltonian mean-field model, a scalar field with quartic interaction, a 1- $d$ self-gravitating system, and a self-gravitating ring.
\end{abstract}

DOI: 10.1103/PhysRevResearch.2.023379

\section{INTRODUCTION}

Long-range interactions, whose potential energy decays with the distance $r$ between interacting bodies slower than $r^{-d}$, where $d$ is the dimension of space [1,2], are relevant to astrophysics and plasma physics $[3,4]$, since gravitational and unscreened Coulomb forces are long-ranged, as well as to condensed matter, given that dipolar interactions in $d=3$ or effective interactions between cold atoms in an electromagnetic cavity [5,6] are long-ranged and occur also in two-dimensional fluids [7]. Systems with long-range interactions exhibit peculiar features both at equilibrium and out of equilibrium $[1,2,8,9]$. In systems with long-range interactions, the dynamics is dominated by collective effects, rather than by binary collisions; as a consequence, the relaxation time towards equilibrium $\tau_{\mathrm{R}}$ diverges with the number of particles $N[3,10]$. In the $N \rightarrow \infty$ limit, or for times $t<\tau_{\mathrm{R}}$ for finite $N$, the dynamics obeys the noncollisional Boltzmann, or Vlasov, equation [1-3]. Introducing the single-particle Hamiltonian for $N$ particles (for simplicity, we assume identical particles with unit masses),

$$
H=\frac{p^{2}}{2}+U(q, t)
$$

\footnotetext{
*ggiachet@sissa.it

†alessandro.santini2@stud.unifi.it

¥lapo.casetti@unifi.it
}

Published by the American Physical Society under the terms of the Creative Commons Attribution 4.0 International license. Further distribution of this work must maintain attribution to the author(s) and the published article's title, journal citation, and DOI. where $q=\left(q_{1}, \ldots, q_{d}\right), p=\left(p_{1}, \ldots, p_{d}\right), U$ is the selfconsistent potential

$$
U(q, t)=\int d p^{\prime} d q^{\prime} f\left(q^{\prime}, p^{\prime}, t\right) V\left(\left|q-q^{\prime}\right|\right),
$$

$f(q, p, t)$ is the single-particle distribution function, and $V(r)$ is the potential energy between two particles at distance $r$, we can write the Vlasov equation as

$$
\frac{\partial f}{\partial t}=\{H, f\},
$$

where $\{\cdot, \cdot\}$ is the Poisson bracket, thus making explicit its symplectic structure. This has important consequences [11-13], e.g., the Vlasov equation is time reversal invariant and its dynamics is constrained by an infinite number of conservation laws: the Casimirs

$$
\mathcal{C}[f]=\int d q d p C(f)
$$

are conserved for any choice of $C(f)$. Remarkably, also the Boltzmann entropy is a Casimir, corresponding to $C(f)=$ $-f \ln f$, so that it is a constant of motion and no $H$ theorem holds. All these properties seem to suggest that no relaxational dynamics is possible: any time dependence of $f$ should survive forever in the $N \rightarrow \infty$ limit and at least up to $t \approx \tau_{\mathrm{R}}$ when collisional effects set in for a large but finite system. Numerical results depict a totally different scenario: starting from a generic initial condition, a given observable exhibits oscillations that damp out on a rather fast time scale not dependent on $N$ (at variance with $\tau_{\mathrm{R}}$ ) until it attains a nearly constant value. The paradigmatic example is gravitational collapse [14-16], where the relevant observable is either the gravitational radius or the virial ratio, so that the damped oscillations are termed "virial oscillations," and this noncollisional relaxation is referred to as "violent relaxation" [17]. Violent relaxation is a universal phenomenon, occurring in 
any long-range-interacting system; the state reached after violent relaxation is referred to as a quasistationary state, may be very far from thermal equilibrium [1,18-21], and in a finite system will eventually relax to equilibrium for $t>\tau_{\mathrm{R}}$. Despite many advances $[1,2,8]$ a theory able to predict these states given a generic initial condition is still missing. It is widely believed that the mechanism of violent relaxation is similar to Landau damping $[13,22,23]$. Basically this means that the Vlasov dynamics never actually stops: rather it trickles down towards smaller and smaller scales until it no longer affects the behavior of any coarse-grained observable. Indeed, given a coarse-grained distribution function $\tilde{f}$, obtained by averaging $f$ over some finite volume $\Delta \Gamma$ in phase space, and any convex function $C(x)$, the corresponding Casimir $\mathcal{C}[\tilde{f}]$ decreases in time [24]. Despite this, a convincing quantitative picture of this process is still missing: our aim is thus to contribute to filling this gap by providing an effective evolution equation for $\tilde{f}$.

The paper is organized as follows. In Sec. II we propose a general form of the effective evolution equation, up to coefficients, based on symmetry considerations only. Then, in Sec. III we explicitly perform the coarse graining and derive the complete equation in the one-dimensional case. Section IV is devoted to predicting the dependence of damping times on the coarse-graining scale and checking the results against numerical simulations of some one-dimensional models: the Hamiltonian mean-field (HMF) model, a scalar field with quartic interaction, a $1-d$ self-gravitating system, and a selfgravitating ring (SGR). Finally, in Sec. V we comment on the results we have obtained and discuss their relation to other approaches, open problems, and future developments. To ease the reading, some proofs and some further details on the numerics are reported in Appendixes A-C.

\section{SYMPLECTIC COARSE GRAINING}

Many properties of an effective evolution equation for $\tilde{f}$ can be derived from symmetry considerations and very general assumptions, which define what we refer to as symplectic coarse graining. First, if $\tilde{f}$ is normalized to unity, then a coarse-grained single-particle Hamiltonian $H[\tilde{f}]$ is defined as in Eq. (1), with $\tilde{f}$ in place of $f$; to ease the notation, we simply write $H$ in place of $H[\tilde{f}]$. We then assume that the coarsegraining procedure does not depend on the choice of the canonical coordinates, preserving the symplectic structure; therefore, the dynamical evolution of $\tilde{f}$ can be expressed in terms of Poisson brackets. Moreover, we assume that Poisson brackets contain functions of $H$ and $\tilde{f}$ alone and are linear in $\tilde{f}$; physically, this means that particles interact only via $H$, as in the Vlasov equation, (3). These assumptions imply that

$$
\frac{\partial \tilde{f}}{\partial t}=\mathcal{L}_{H}(\tilde{f})
$$

where $\mathcal{L}_{H}(\tilde{f})$ depends on $H$, it acts linearly on $\tilde{f}$, and its most general form is a linear combination of nested Poisson brackets where $\tilde{f}$ appears only once, that is, of terms of the form $\left\{\lambda_{1}(H),\left\{\lambda_{2}(H),\left\{\ldots\left\{\lambda_{k}(H), \tilde{f}\right\} \ldots\right\}\right\}\right\}$, where the $\lambda_{k}$ 's are generic functions of $H$. By repeatedly using the identity $\left\{\lambda_{k}(H), \cdot\right\}=\lambda_{k}^{\prime}(H)\{H, \cdot\}$ and denoting by $\{H, \cdot\}^{n} \tilde{f}$ the $n$ nested Poisson brackets, i.e.,

$$
\{H, \cdot\}^{n} \tilde{f}=\underbrace{\{H,\{H,\{\ldots\{H, \tilde{f}\} \ldots\}\}\}}_{n \text { times }},
$$

we can thus write

$$
\frac{\partial \tilde{f}}{\partial t}=\{H, \tilde{f}\}+\sum_{n=2}^{\infty} \mu_{n}(H)\{H, \cdot\}^{n} \tilde{f},
$$

where the $\mu_{n}$ 's are generic functions that absorb the coefficients of the linear combination. In Eq. (7) we have highlighted the first term of the sum, assuming $\mu_{1}(H) \equiv 1$, as is reasonable since $\tilde{f} \rightarrow f$ and Eq. (7) must reduce to Eq. (3) when ${ }^{1} \Delta \Gamma \rightarrow 0$. We note that both the normalization of $\tilde{f}$ and the total energy $E[\tilde{f}]$ are conserved by Eq. (7), as required by a physically sound evolution (see Appendix A1).

Equation (7) is the most general outcome of symplectic coarse graining. The terms of the sum on the right-hand side (r.h.s.) of Eq. (7) containing an odd number of Poisson brackets do not break time-reversal invariance, so that they renormalize the time-reversible Vlasov evolution, while those containing an even number of brackets break the time-reversal invariance and may account for dissipation. However, we expect that not all the possible $\mu_{n}$ 's are physically admissible. For instance, as already mentioned, all the convex Casimirs defined by the coarse-grained distribution function $\tilde{f}$ must decrease with time. It is not easy to impose such a constraint on Eq. (7), but the lowest-order truncation of the latter equation, obtained by setting $\mu_{n}=0 \forall n>2$,

$$
\frac{\partial \tilde{f}}{\partial t}=\{H, \tilde{f}\}+\mu_{2}(H)\{H,\{H, \tilde{f}\}\},
$$

with the additional constraint $\mu_{2}(x)>0 \forall x$, does satisfy this constraint (see Appendix A 2 a) and actually describes a Vlasov-like evolution with added diffusive effects, hence admitting a relaxational behavior. Indeed, $\{H, \cdot\}$ is proportional to the directional derivative along the Hamiltonian flow generated by $H$, so that $\{H,\{H, \cdot\}\}$ is a sort of anisotropic Laplacian and the second term on the r.h.s. of Eq. (8) describes a diffusion taking place along the Hamiltonian flow, whose strength depends on $\mu_{2}$, which in turn will depend on the coarse-graining scale $\Delta \Gamma$; this will become apparent in the one-dimensional case that we tackle in the following. Once $\Delta \Gamma$ is fixed, Eqs. (7) and (8) are expected to be appropriate to describe the evolution of an observable which is not sensitive to the structure of $f$ on scales smaller than $\Delta \Gamma$ itself. Choosing as $\Delta \Gamma$ the smallest scale the observable of interest is sensitive to, the odd (conservative) terms in Eqs. (7) and (8) will eventually relocate the dynamics on scales smaller than $\Delta \Gamma$, while the even (dissipative) terms will erase such information in $\tilde{f}$, thus effectively describing the dynamics of the chosen observable.

\footnotetext{
${ }^{1}$ We implicitly assume that the sum of the contributions to Eq. (7) with $n \geqslant 2$ vanishes when $\Delta \Gamma \rightarrow 0$.
} 


\section{EFFECTIVE EQUATION FOR ONE-DIMENSIONAL SYSTEMS}

Let us perform a symplectic coarse graining and obtain an explicit evolution equation for the coarse-grained $\tilde{f}$ in the case of 1- $d$ systems, bounded in space or with periodic boundary conditions. In this case $H$ has 1 degree of freedom, so that, at a given time, it is integrable and a canonical transformation, $(p, q) \mapsto(J, \vartheta)$, exists, where $(J, \vartheta)$ are actionangle variables. $H$ being time dependent in general, such a transformation leads to a Hamiltonian independent of the angle $\vartheta$ only at a given time $t$. The instantaneous flow will be such as to keep $J$ constant, and the angle will linearly evolve in time,

$$
\vartheta(t+\Delta t)=\vartheta(t)+\omega(J) \Delta t
$$

where $\omega(J)=d H / d J$. Let us consider a (small) interval of actions $\Delta J=J_{2}-J_{1}$, define $\bar{\omega}$ as the frequency $\omega$ averaged over $\Delta J$,

$$
\bar{\omega}=\frac{1}{\Delta J} \int_{J_{1}}^{J_{2}} \omega\left(J^{\prime}\right) d J^{\prime},
$$

and consequently $\delta \omega=\omega-\bar{\omega}$, and a distribution function coarse-grained along $J$ as

$$
\bar{f}(J, \vartheta)=\frac{1}{\Delta J} \int_{J_{1}}^{J_{2}} f\left(J^{\prime}, \vartheta\right) d J^{\prime},
$$

where $J$ is such that $\bar{\omega}=\omega(J)$. To get a truly coarse-grained distribution function one should average also over an interval of angles $\Delta \vartheta$, but it is more convenient to consider such an average as carried over a time interval $\Delta t$, that is, to assume that we are blind to changes of the coordinates of the particles occurring on time scales smaller than $\Delta t$. This means that we neglect the time dependence of $H$ on a time scale $\Delta t$, and we can use action-angle coordinates for times between $t$ and $t+\Delta t$, defining a nonconstant coarse-graining scale on $\vartheta$, namely, $\Delta \vartheta=\bar{\omega} \Delta t$. Our coarse-grained distribution function $\tilde{f}$ is then the function $\bar{f}$ given by Eq. (11), further averaged over an interval of angles of width $\Delta \vartheta$ centered in $\vartheta$. As a consequence, we are not able to distinguish any change of $\tilde{f}$ on scales smaller than $\Delta \Gamma=\Delta \vartheta \Delta J$. For times between $t$ and $t+\Delta t$ we have approximated the flow in phase space with a stationary one, so that its evolution operator should be written as

$$
U_{\Delta t}=e^{\Delta t\{H, \cdot\}} .
$$

The latter is not constant over $\Delta \Gamma$, but within this volume we can consider $J$ and $\vartheta$ as uniformly distributed random variables, so that (up to very unlikely initial conditions) we can write

$$
\tilde{f}_{t+\Delta t}=\tilde{U}_{\Delta t} \tilde{f}_{t},
$$

where we have replaced the evolution operator $U_{\Delta t}$ with the coarse-grained one,

$$
\tilde{U}_{\Delta t}=\left\langle e^{\Delta t\{H, \cdot\}}\right\rangle_{\Delta \Gamma} .
$$

The evolution dictated by Eq. (14) satisfies the constraint on the evolution of convex Casimirs (see Appendix A2b) and can be translated into a differential equation for the coarse-grained distribution function $\tilde{f}(t)$. To derive this equation, we start by writing the evolution operator in action-angle variables,

$$
\tilde{U}_{\Delta t}=\left\langle e^{\Delta t\{H, \cdot\}}\right\rangle_{\Delta \Gamma}=\left\langle e^{-\omega\left(J^{\prime}\right) \Delta t \partial_{\vartheta}}\right\rangle_{\Delta J} .
$$

Then, since operators at the exponent evaluated at different points commute, we can apply the usual cumulant expansion and find

$$
\tilde{U}_{\Delta t}=\exp \left[\sum_{n=1}^{\infty} \frac{(-\Delta t)^{n}}{n !} \kappa_{n}(\omega) \partial_{\vartheta}^{n}\right],
$$

where $\kappa_{n}$ is the $n$th cumulant of the probability distribution of the frequencies $\omega$. The time evolution becomes

$$
\tilde{f}_{t+\Delta t}=\exp \left[\Delta t \partial_{t}\right] \tilde{f}_{t}=\exp \left[\sum_{n=1}^{\infty} \frac{(-\Delta t)^{n}}{n !} \kappa_{n}(\omega) \partial_{\vartheta}^{n}\right] \tilde{f}_{t},
$$

so that

$$
\frac{\partial \tilde{f}}{\partial t}=\sum_{n=1}^{\infty}(-1)^{n} \frac{\kappa_{n}(\omega)(\Delta t)^{n-1}}{n !} \frac{\partial^{n} \tilde{f}}{\partial \vartheta^{n}} .
$$

Let $\delta \omega=\omega-\bar{\omega}$, where $\bar{\omega}=\omega(J)=\kappa_{1}(\omega)$ is the average of the distribution of the frequencies $\omega$. Then $\kappa_{1}(\delta \omega)=0$ and $\kappa_{n}(\delta \omega)=\kappa_{n}(\omega)$ for any $n>1$, so that we can extract the first term from the sum in Eq. (18) and obtain

$$
\frac{\partial \tilde{f}}{\partial t}=-\omega(J) \frac{\partial \tilde{f}}{\partial \vartheta}+\sum_{n=2}^{\infty}(-1)^{n} D_{n}(J) \frac{\partial^{n}}{\partial \vartheta^{n}} \tilde{f},
$$

where we have introduced the diffusion coefficients

$$
D_{n}(J)=\frac{(\Delta t)^{n-1}}{n !} \kappa_{n}(\delta \omega) .
$$

Equations (19) and (20) are written as such only at the time $t$ chosen to define the action-angle coordinates. However, we can rewrite the equation in a coordinate-independent way, by noting that $-\omega(J) \partial_{\vartheta}=\{H, \cdot\}$, so that Eq. (19) is nothing but Eq. (7) with the coefficients $\mu_{n}(H)$ explicitly given ${ }^{2}$ as

$$
\mu_{n}=D_{n}(J(H))[\omega(J(H))]^{-n} .
$$

Note that Eq. (20) implies that all the diffusion coefficients vanish if $\omega$ does not depend on $J$ : in this case $\tilde{f}$ obeys the Vlasov equation as the fine-grained $f$ does. This is coherent with our picture, because no randomness is present if $\omega$ does not depend on $J$ and all the particles coherently drift in $\vartheta$ at the same frequency. Indeed, in the harmonic case where $H$ is linear in $J$ and $\omega$ is constant the motion can be described in terms of normal coordinates without any damping. As already discussed, the even terms are those responsible for the breaking of the time-reversal symmetry; we can estimate their order of magnitude as

$$
D_{2 n} \propto \frac{(\Delta J)^{2 n}(\Delta t)^{2 n-1}}{2^{2 n}(2 n+1) !},
$$

while the odd coefficients are even more suppressed with $n$, the distribution of $\delta \omega$ being even at the leading order. Hence,

\footnotetext{
${ }^{2}$ Being that, at a given time $t, H=H(J)$, the action variable $J$ is implicitly a function of $H$.
} 
as long as $\Delta J$ and $\Delta t$ are not too large, only the very first terms of the sum in Eq. (19) will give a nonnegligible contribution. To leading order in $\Delta J$ and $\Delta t$ the evolution equation for $\tilde{f}$ becomes a Fokker-Planck equation. Retaining only the lowestorder terms in Eq. (19) we can write $\delta \omega(y)=\omega^{\prime}(J)(y-J)$, where $y$ is a random action uniformly distributed between $J-\frac{1}{2} \Delta J$ and $J+\frac{1}{2} \Delta J$; then $\delta \omega$ is uniformly distributed in the interval $\left[-\frac{1}{2}\left|\omega^{\prime}(J)\right| \Delta J, \frac{1}{2}\left|\omega^{\prime}(J)\right| \Delta J\right]$ so that, denoting by $D_{n}^{0}(J)$ the leading-order approximation of the diffusion coefficients, we have

$$
D_{2}^{0}(J)=\frac{\Delta t}{2} \kappa_{2}(\delta \omega)=\frac{1}{24}\left[\omega^{\prime}(J) \Delta J\right]^{2} \Delta t,
$$

and the lowest-order truncation of Eq. (19) can be written as

$$
\frac{\partial \tilde{f}}{\partial t}=-\omega(J) \frac{\partial \tilde{f}}{\partial \vartheta}+D_{2}^{0}(J) \frac{\partial^{2} \tilde{f}}{\partial \vartheta^{2}},
$$

where

$$
D_{2}^{0}(J)=\Delta t \frac{\left[\omega^{\prime}(J) \Delta J\right]^{2}}{24}
$$

and $\omega^{\prime}=d \omega / d J$. Being that $\mu_{2}=D_{2}^{0}(J) \omega^{-2}$ and $(d \omega / d J)^{2} \omega^{-2}=(d \omega / d H)^{2}$, Eq. (24) can be cast in the covariant form

$$
\frac{\partial \tilde{f}}{\partial t}=\{H, \tilde{f}\}+\frac{1}{24} \Delta t(\Delta J)^{2}\{\omega(H),\{\omega(H), \tilde{f}\}\},
$$

which is a special case of Eq. (8). Equation (26) can be interpreted in the corresponding Langevin formalism (see Appendix B). Note that we have taken advantage of the existence of action-angle coordinates (at least at a given time) to derive our results, but then we have expressed them in a covariant fashion, so that they do not depend on the choice of coordinates.

\section{SCALING OF DAMPING TIMES} $\tilde{f}$ is

According to Eq. (24), the characteristic damping time of

$$
\tau=\left(D_{2}^{0}\right)^{-1} .
$$

Let us now ask how $\tau$ depends on the coarse-graining scale. In order for our coarse graining to be independent of the choice of the coordinates in phase space we have to define its scale in terms of phase-space volumes (i.e., surfaces since $d=1$ ), which are invariant under canonical transformations. Let $\Delta \Gamma=\Delta J \Delta \vartheta$, so that we may assume that $\Delta J \propto \sqrt{\Delta \Gamma}$ and $\Delta \vartheta \propto \sqrt{\Delta \Gamma}$. Equation (25) contains $\Delta t$ instead of $\Delta \vartheta$, because of the way we have performed the symplectic coarse graining; however, $\Delta t \propto \Delta \vartheta \propto \sqrt{\Delta \Gamma}$, so that Eqs. (25) and (27) imply $\tau \propto(\Delta \Gamma)^{-3 / 2}$. If $S$ is the smallest surface we want to probe, neglecting the dynamics occurring on scales smaller that $S$, we have to choose $\Delta \Gamma \approx S$, so that the damping time at scale $S$ obeys the scaling relation

$$
\tau \propto S^{-3 / 2} .
$$

The time given by (28) is the time after which the dynamics of the fine-grained $f$ has moved to scales smaller than $S$ in phase space, so that our coarse-grained description is no longer able to detect it. One way to probe different scales is to look at the
Fourier components $f_{\mathbf{k}}$ of the distribution function in phase space, where $\mathbf{k}=\left(k_{p}, k_{q}\right)$, with $k_{q}$ and $k_{p}$ its components along any couple of canonical coordinates $(p, q)$,

$$
f(\mathbf{k}, t)=\int e^{i\left(k_{p} p+k_{q} q\right)} f(p, q, t) d p d q ;
$$

a given $f_{\mathbf{k}}$ probes a strip in phase space of a width proportional to $k^{-1}$, where $k=\left(k_{p}^{2}+k_{q}^{2}\right)^{1 / 2}$. Therefore, to describe the evolution of $f_{\mathbf{k}}$ we have to choose $S \propto k^{-1}$, and we expect $f_{\mathbf{k}}$ to damp out on the time scale

$$
\tau_{\mathbf{k}} \propto k^{3 / 2} .
$$

\section{Numerical results}

To check the scaling law, (30), we solved the Vlasov equation for various one-dimensional models on an $N_{q} \times N_{p}$ grid with a time step $\Delta t$ until a maximum time $t=t_{\max }$ using a semi-Lagrangian method [25,26], computing the evolution of the $f_{\mathbf{k}}$ starting from nonstationary configurations, and always finding good agreement between Eq. (30) and numerics. Results are reported in the following subsections, while the protocol to measure the damping times $\tau_{\mathbf{k}}$ is described in Appendix C.

\section{Hamiltonian mean-field model}

The HMF model [27] has been the workhorse of studies on long-range-interacting systems in the last decades. The Hamiltonian of the model is

$$
H=\sum_{i=1}^{N} \frac{p_{i}^{2}}{2}-\frac{J}{2 N} \sum_{i=1}^{N} \sum_{j=1}^{N} \cos \left(q_{i}-q_{j}\right),
$$

where $q_{i} \in[-\pi, \pi]$ and $p_{i} \in \mathbb{R}$, for $i=1, \ldots, N$, are canonically conjugated coordinates. This model can be seen either as a system of globally coupled $X Y$ spins or as $N$ particles with unit mass moving on a ring interacting via a cosine potential. In the following we use natural units to obtain dimensionless quantities, setting $J=1$, thus considering only attractive (ferromagnetic, in the spin language) interactions. In the limit $N \rightarrow \infty$, the dynamics of the oneparticle distribution function $f(q, p, t)$ is given by the Vlasov equation

$$
\frac{\partial f}{\partial t}+p \frac{\partial f}{\partial q}-\frac{d V[f]}{d q} \frac{\partial f}{\partial p}=0,
$$

where

$$
\begin{aligned}
V[f](q) & =-m_{x}[f] \cos q-m_{y}[f] \sin q, \\
m_{x}[f] & =\int d q d p f \cos q, \\
m_{y}[f] & =\int d q d p f \sin q .
\end{aligned}
$$

In Fig. 1 we show $\tau_{\mathbf{k}}$ as a function of $\mathbf{k}$ for $0<k \lesssim 120$ and the same numbers rescaled according to Eq. (30), for "waterbag" initial conditions, i.e., $q$ 's and $p$ 's drawn from a uniform distribution with compact support. While the $\tau_{\mathbf{k}}$ 's span three orders of magnitude, almost all the rescaled times are $\mathcal{O}(1)$. Damping times depend on the initial conditions: as 


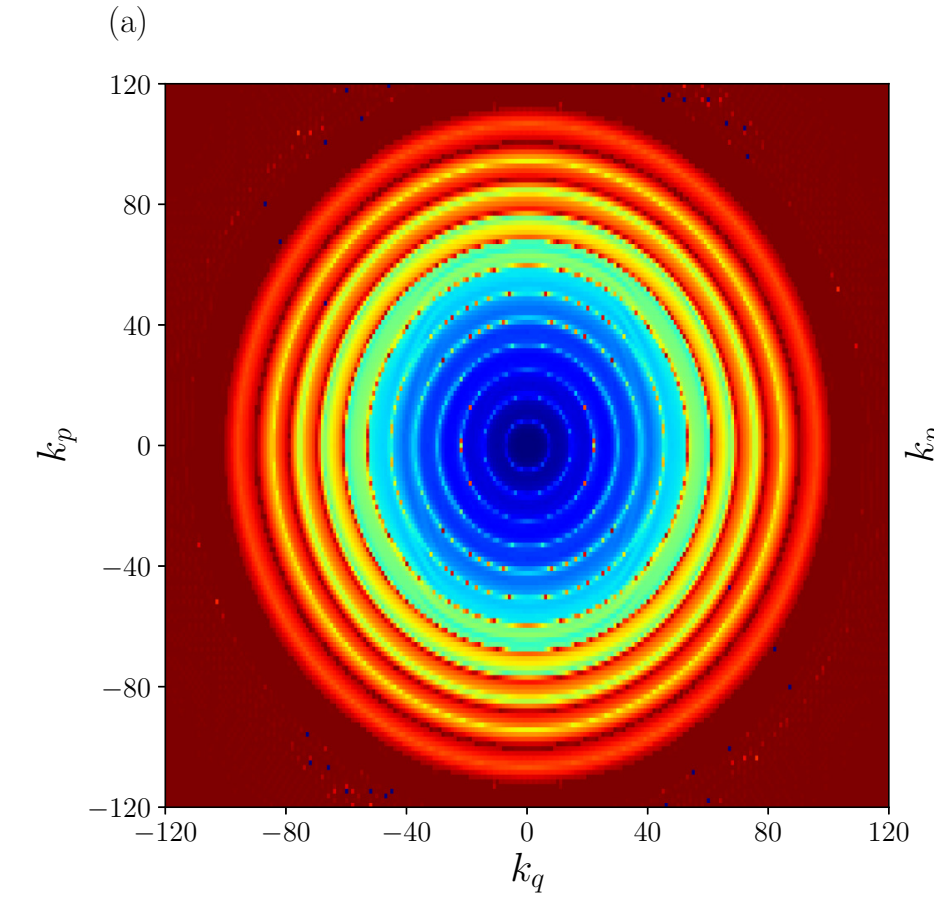

(b)
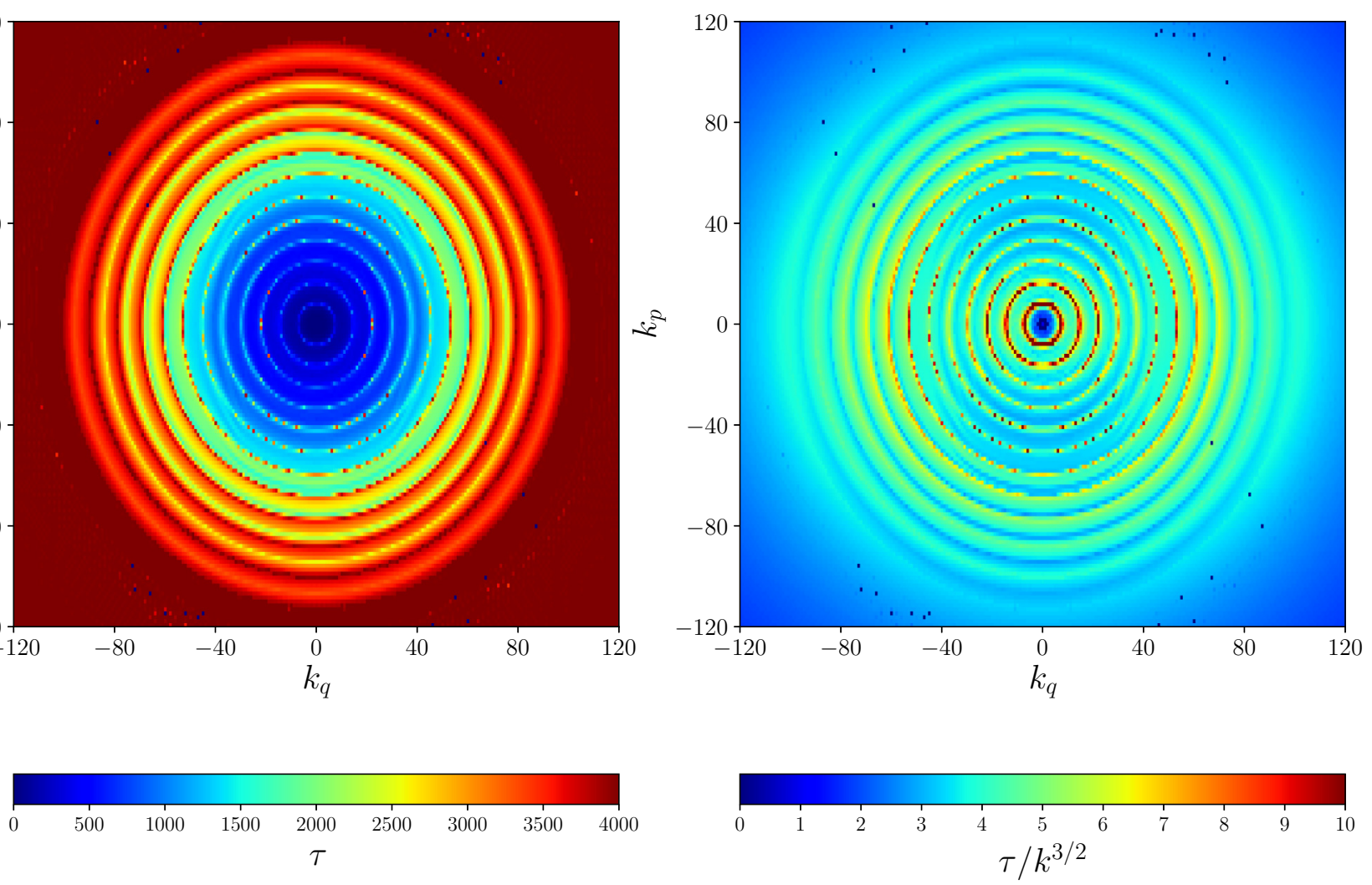

FIG. 1. HMF model: damping times of the Fourier components $f_{\mathbf{k}}$ of the distribution function defined in Eq. (29). (a) Damping times as a function of $\mathbf{k}$. (b) Rescaled damping times $\tau_{\mathbf{k}} / k^{3 / 2}$. Note the difference in scale between (a) and (b). The Vlasov equation was solved on an $N_{q} \times N_{p}=1064 \times 1248$ grid with a time step $\Delta t=2.5 \times 10^{-3}$ until $t_{\max }=4 \times 10^{3}$. Initial conditions: $q$ 's and $p$ 's uniformly distributed in $[-\pi / 2, \pi / 2]$ and $[-0.25,0.25]$, respectively.

an example, in Fig. 2 we show damping times and rescaled damping times obtained starting from different initial conditions with respect to the case shown in Fig. 1: here, positions are still drawn from a uniform distribution with compact support, but now the momenta are drawn from a Gaussian distribution. The scaling law, (30), is in good agreement with the data, although here the interval over which the rescaled damping times are distributed is larger than in the previous case (nonetheless, it is still $6 \times 10^{-3}$ times the interval of the values of the computed damping times).

In the following we consider three other models living in one dimension: a one-dimensional scalar field interacting via a mean-field quartic potential, ${ }^{3}$ a one-dimensional selfgravitating system, and the so-called self-gravitating ring model. All the models being one-dimensional, the Vlasov equation is of the form of (32) for all of them, but the self-consistent interaction potential $V[f(q)]$ will be different for each model. The initial conditions will be the same in all the examples we consider and will be equal to those considered for the HMF model in the example reported in Fig. 2, i.e., uniform on the segment $[-1,1]$ for the coordinates

\footnotetext{
${ }^{3}$ Note that the interaction in this model is not periodic in the coordinates, at variance with all the other models.
}

and Gaussian, with zero mean and standard deviation equal to 0.1 , for the momenta.

\section{Scalar field with mean-field quartic interaction}

The mean-field-interacting scalar-field model can be seen as the continuum limit of the $N$-particle Hamiltonian

$$
H=\sum_{i=1}^{N} \frac{p_{i}^{2}}{2}+\frac{1}{2 N} \sum_{i=1}^{N} \sum_{j=1}^{N} \frac{1}{4 !}\left(q_{i}-q_{j}\right)^{4},
$$

where $q_{i} \in \mathbb{R}$ and $p_{i} \in \mathbb{R}$, for $i=1, \ldots, N$, are canonically conjugated coordinates. In the Vlasov limit $N \rightarrow \infty$ selfconsistent interaction potential is

$$
V[f](q)=\int d q^{\prime} d p^{\prime} \frac{\left(q-q^{\prime}\right)^{4}}{4 !} f\left(q^{\prime}, p^{\prime}, t\right) .
$$

An example of computed and rescaled damping times for this model is shown in Fig. 3. The agreement between the numerical data and the scaling law $\tau_{\mathbf{k}} \propto k^{3 / 2}$ is apparently very good.

\section{One-dimensional self-gravitating system}

A one-dimensional self-gravitating system can be seen as $N$ infinite massive parallel planes, with a constant 

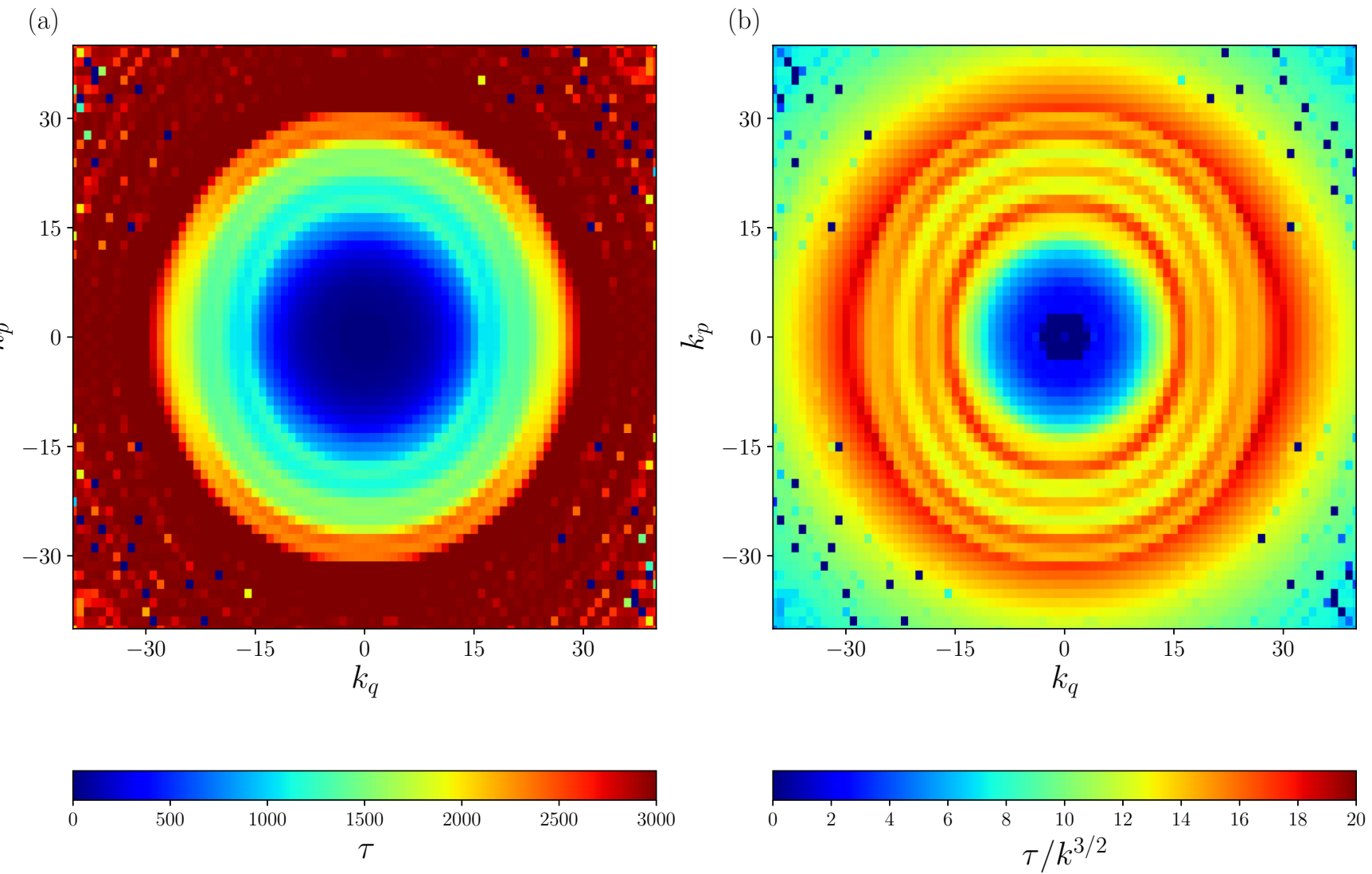

FIG. 2. As Fig. 1, with initial conditions such that the $q$ 's are uniformly distributed in $[-1,1]$ and the $p$ 's are normally distributed with zero mean and standard deviation equal to 0.1 ; other simluation parameters are $N_{q} \times N_{p}=1000 \times 1125, \Delta t=2 \times 10^{-3}$, and $t_{\max }=3 \times 10^{3}$.

surface mass density, moving in the direction orthogonal to the planes themselves. Assuming periodic boundary conditions, the gravitational interaction can be expanded in a Fourier series, so that the Hamiltonian can be written, after introducing dimensionless variables, as

$$
H=\sum_{i=1}^{N} \frac{p_{i}^{2}}{2}-\frac{1}{2} \sum_{n=1}^{\infty} \frac{1}{n^{2}}\left(m_{x, n}^{2}+m_{y, n}^{2}\right)
$$

where $p_{i} \in \mathbb{R}$, for $i=1, \ldots, N$, and

$$
\begin{aligned}
& m_{x, n}=\frac{1}{N} \sum_{i=1}^{N} \cos \left(n q_{i}\right), \\
& m_{y, n}=\frac{1}{N} \sum_{i=1}^{N} \sin \left(n q_{i}\right),
\end{aligned}
$$

with $q_{i} \in[-\pi, \pi]$, for $i=1, \ldots, N$. Hence the selfconsistent potential entering the Vlasov equation for this model is

$$
V[f](q)=-\sum_{n=1}^{+\infty} \frac{1}{n^{2}}\left[m_{x}^{(n)} \cos (n q)+m_{y}^{(n)} \sin (n q)\right],
$$

with

$$
\begin{aligned}
& m_{x}^{(n)}[f]=\int d q d p f(p, q, t) \cos (n q), \\
& m_{y}^{(n)}[f]=\int d q d p f(p, q, t) \sin (n q) .
\end{aligned}
$$

In practice, one can consider a large but finite number $M$ of Fourier modes of the interaction, so that the infinite series in Eqs. (36) and (38) are replaced by finite sums, with $n$ running from 1 to $M$; we considered $M=250$. Note that if we take $M=1$, we get back to the HMF model, whose interaction can then be seen as the lowest-order Fourier approximation of self-gravity in one dimension. An example of computed and rescaled damping times for this model is shown in Fig. 4. Again, the agreement between the numerical data and the scaling law, (30), is very good.

\section{Self-gravitating ring}

Instead of working as in Sec. IV A 3 with low-dimensional gravity, one can consider (softened) three-dimensional gravitational forces but constrain the interacting particles to move on a ring; the resulting model is referred to as the selfgravitating ring, introduced in [28] and further studied in [29-32]. The Hamiltonian, again expressed in dimensionless 
(a)
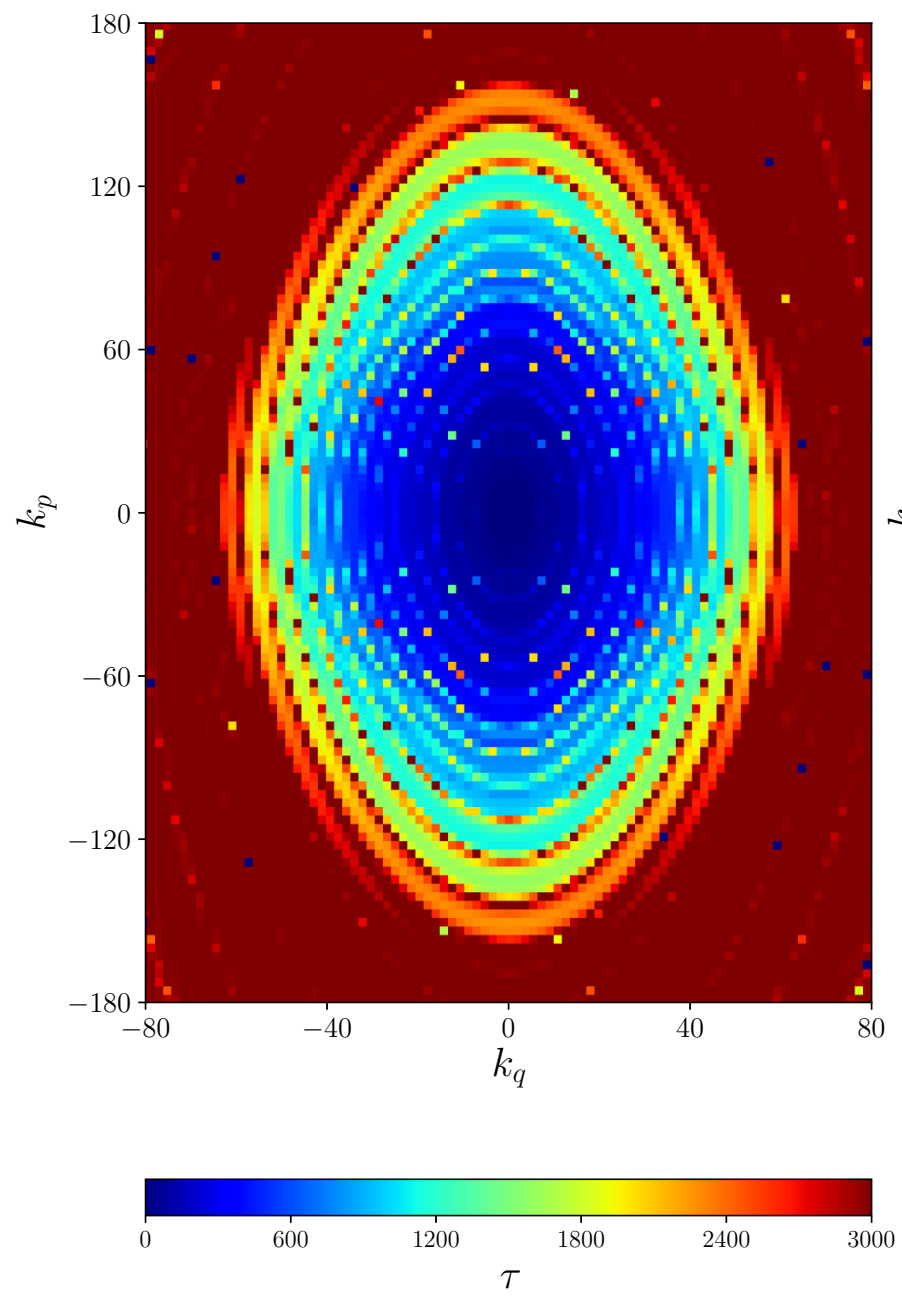

(b)
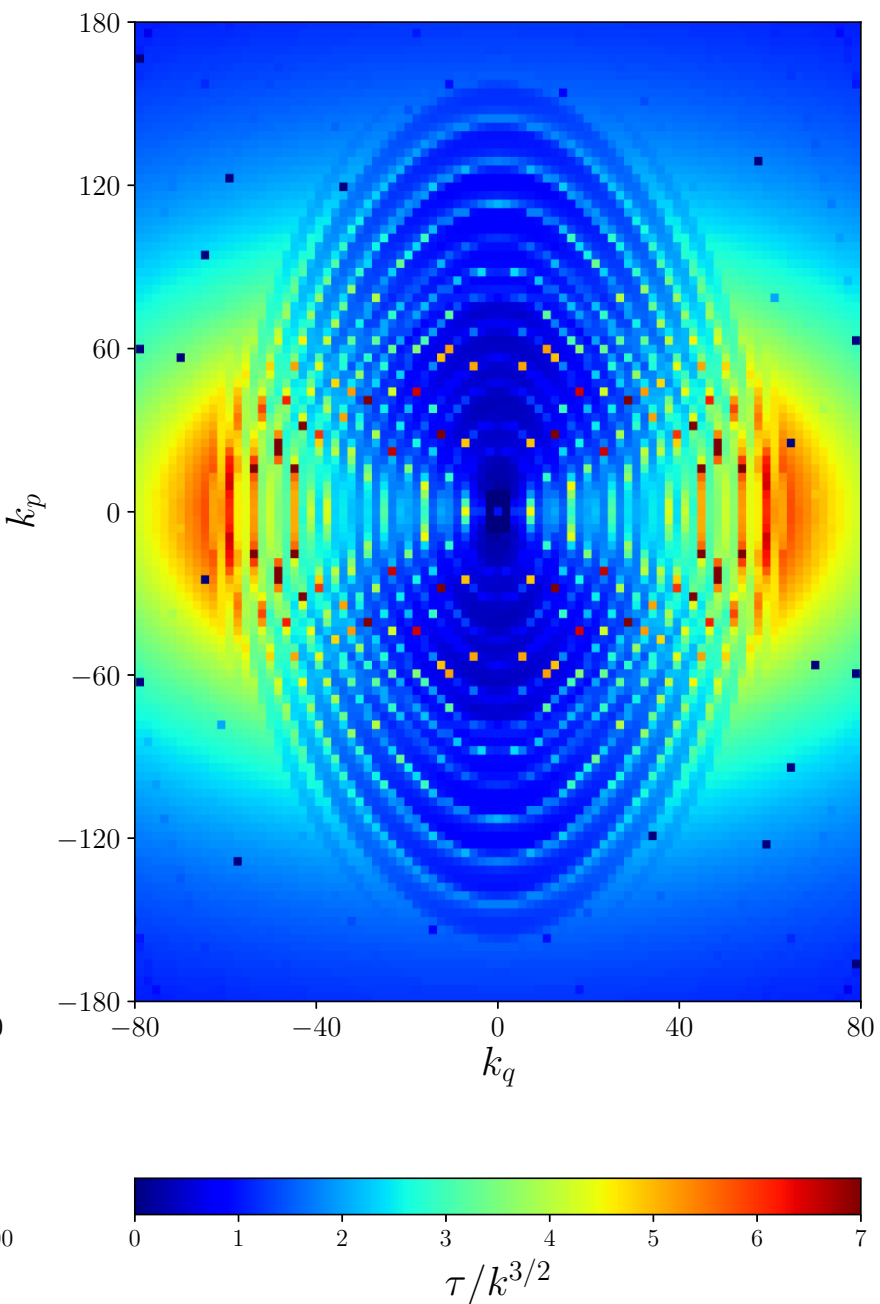

FIG. 3. As Fig. 1, for a scalar field with mean-field quartic interaction. Initial conditions are as in Fig. 2, and other simulation parameters are $N_{q} \times N_{p}=512 \times 1024, \Delta t=10^{-2}$, and $t_{\max }=3 \times 10^{3}$.

variables, is

$$
H=\sum_{i=1}^{N} \frac{p_{i}^{2}}{2}-\frac{1}{2 \sqrt{2} N} \sum_{i=1}^{N} \sum_{j=1}^{N}\left[\frac{1}{\sqrt{1-\cos \left(q_{i}-q_{j}\right)+\alpha}}\right],
$$

where $q_{i} \in[-\pi, \pi]$ and $p_{i} \in \mathbb{R}$, for $i=1, \ldots, N$, are canonically conjugated coordinates and $\alpha$ is a softening parameter, regularizing the divergence of the gravitational interaction for vanishing distance between the particles. It can be shown [29] that the SGR reduces to the HMF in the limit $\alpha \rightarrow \infty$. The self-consistent potential entering the Vlasov equation for this model is written as

$$
V[f](q)=-\frac{1}{\sqrt{2}} \int_{-\pi}^{\pi} d q^{\prime} \int_{-\infty}^{\infty} d p^{\prime} \frac{f\left(q^{\prime}, p^{\prime}, t\right)}{\sqrt{1-\cos \left(q-q^{\prime}\right)+\alpha}} .
$$

This model is somewhat harder to solve numerically than the previous ones, and numerical diffusion prevents reliable results for Fourier component $f_{\mathbf{k}}$ with large $k$ 's, so that we had to limit ourselves to shorter simulations and to damping times corresponding to smaller wave vectors than in the previous cases. This notwithstanding, we are able to see the good agreement between the predicted scaling law $\tau_{\mathbf{k}} \propto k^{3 / 2}$ and the numerical results also for the SGR (see Fig. 5).

\section{CONCLUSIONS}

We have derived an effective evolution equation for a coarse-grained distribution function in the case of systems whose dynamics obeys the Vlasov equation in the $N \rightarrow \infty$ limit. A general form of the equation has been given based on symmetry considerations, i.e., requiring the conservation of the symplectic structure, and an explicit equation for 1-d systems was derived independently of the general equation: the fact that we indeed found an equation of the same form as the general one is a nontrivial result and supports the validity of our approach. The lowest-order term of the equation is a diffusion along the Hamiltonian flow and becomes, if $f$ is stationary, a diffusion along the $J=$ constant lines in phase space. Diffusion in the stationary case (which implies a dynamics analogous to that dictated by a fixed external potential) is due to the dependence on $J$ of the frequency $\omega$, which 
(a)
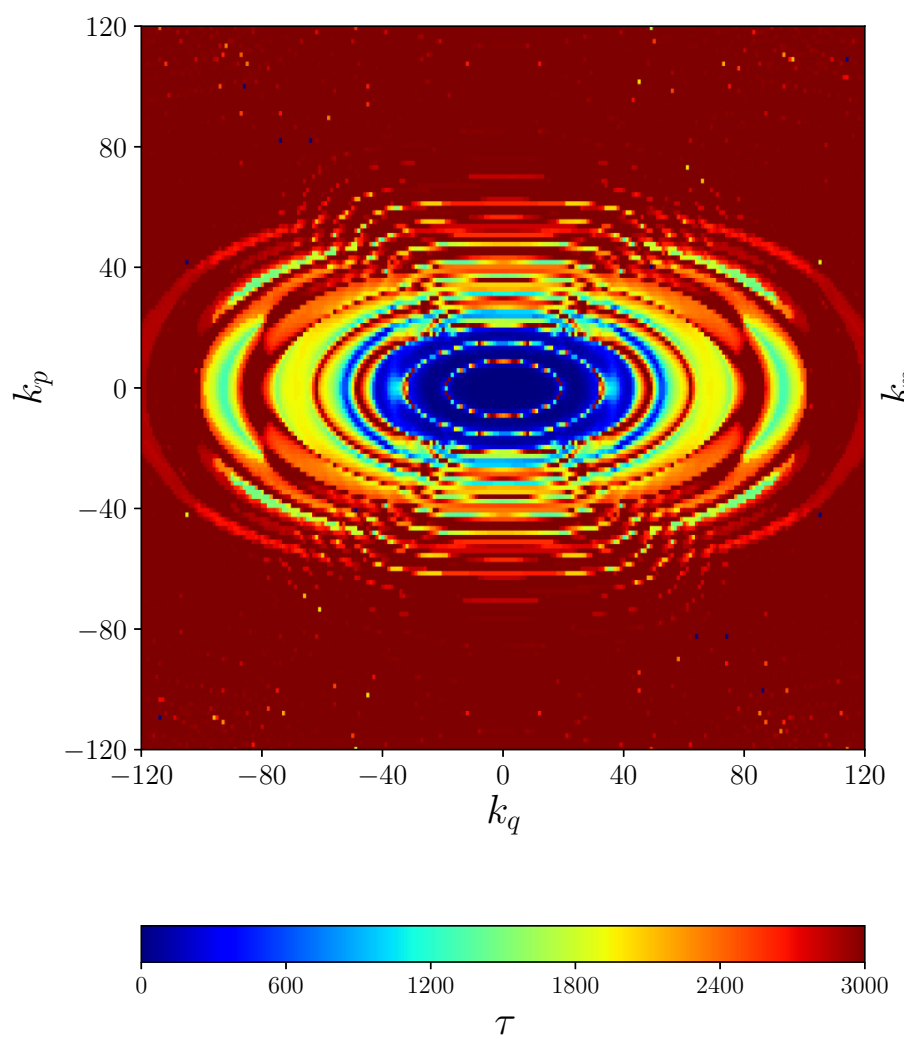

(b)
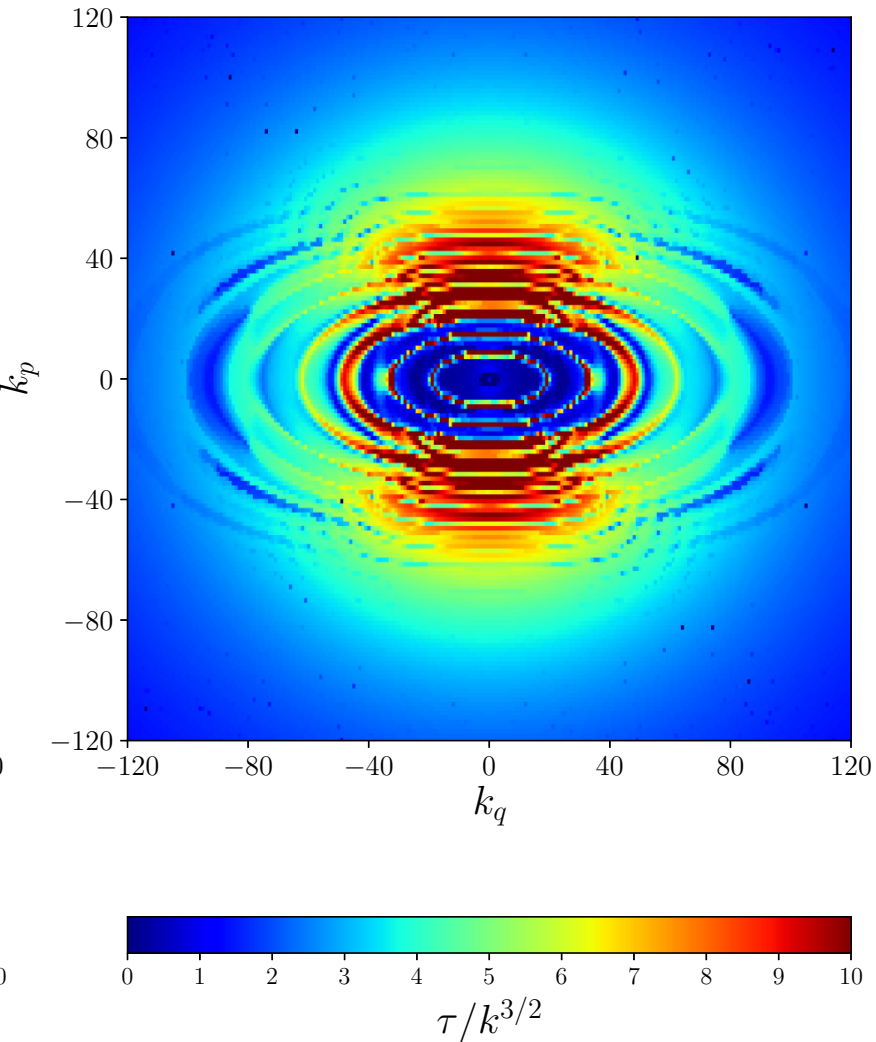

FIG. 4. As Fig. 1, for a one-dimensional self-gravitating system with periodic boundary conditions. Initial conditions are as in Fig. 2 , and other simulation parameters are $N_{q} \times N_{p}=1024 \times 1024, \Delta t=2 \times 10^{-3}$, and $t_{\max }=3 \times 10^{3}$.

entails differential rotation in phase space, a filamentation of $f$, and thus an effective mixing in phase space due to our blindness to small scales after coarse graining. Indeed, and as it should be, if $\omega$ does not depend on $J$ as in the harmonic case, no diffusion is present in our theory. Depending on $\omega$, diffusion may be either very effective (as happens close to separatices) or not efficient at all. In the latter case our theory may predict long-standing oscillations, which may be an alternative outcome of violent relaxation instead of damping to a quasistationary state [33-36]. Diffusion along equal action lines has been shown to be effective for the HMF model in the stationary case [37]: the latter results are an independent, indirect check of the soundness of our approach. Our results provide a solid quantitative picture of the mechanism underlying violent relaxation and shed light on the rôle of the coarse-graining scale: numerical results for $1-d$ systems are in very good agreement with our predictions.

We note that an effective equation with the same kind of structure as the one presented here has been found for suitable moments of the distribution function, at leading order and based on heuristic considerations, in [21]. An effective description that eliminates the small-scale Vlasov dynamics appears in [38] and [39], where a phenomenological maximum entropy production principle is invoked to get a diffusion in velocity space, which apparently does not conserve the symplectic structure, at variance with our approach. In [40] a deterministic coarse-graining procedure was introduced, yielding a time-reversal-invariant effective evolution, at vari- ance with the one we have derived here. As argued in [41], a faster-than-collisional relaxation might also be induced by a finite number of particles $N$; however, such a mechanism seems not to be relevant to violent relaxation, which occurs in finite systems as well as in the Vlasov $N \rightarrow \infty$ limit and whose time scale does not depend on $N$. Although we have explicitly derived the effective equation only in the 1-d case, we expect the extension of our procedure to systems with $d>1$ that are integrable at a given time, e.g., the self-gravitating case with imposed spherical symmetry, to be possible. Moreover, one may think of applying a truncated form of the general equation, e.g., Eq. (8), supplemented by some ansatz for the unknown function $\mu_{2}$, to describe violent relaxation in generic long-range-interacting systems.

\section{ACKNOWLEDGMENTS}

This work is part of MIUR-PRIN2017 Project No. 201798CZL, "Coarse-grained description for non-equilibrium systems and transport phenomena (CO-NEST)," whose partial financial support is acknowledged.

\section{APPENDIX A: PROOFS OF SOME ANALYTICAL RESULTS}

We present here proofs of some results put forward in the paper. First, let us recall some properties of Poisson brackets. We consider a Hamiltonian system with $d$ degrees of 
(a)
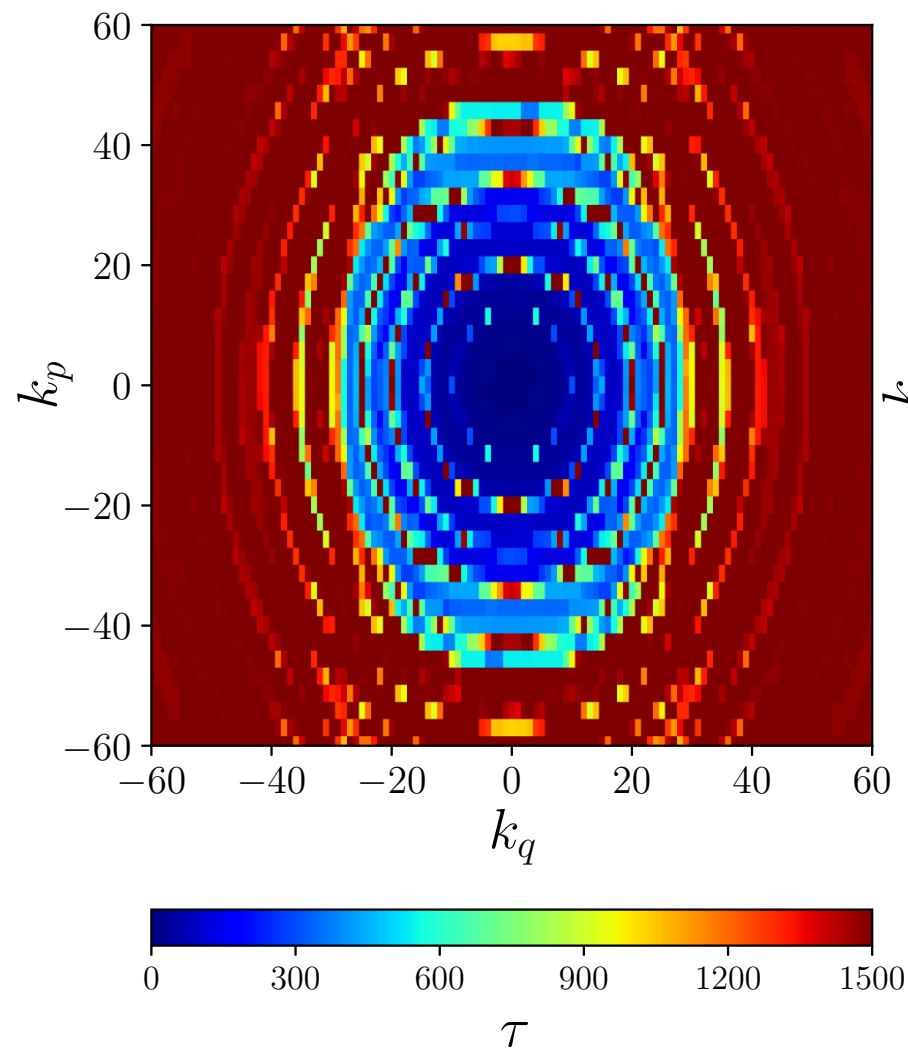

(b)

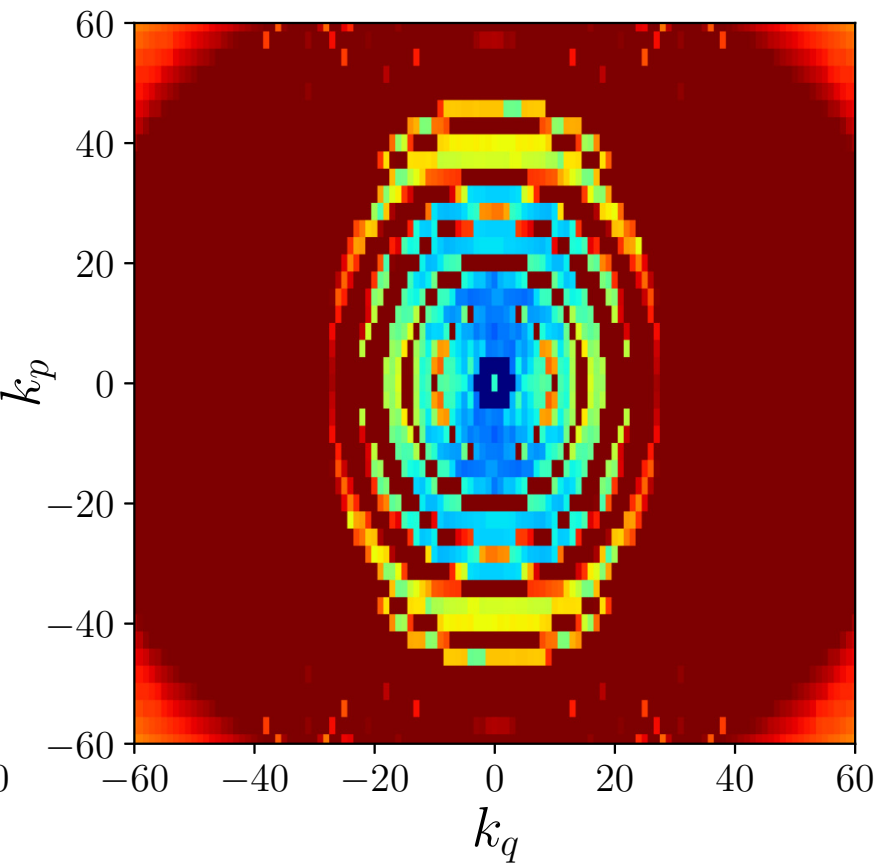

0.0

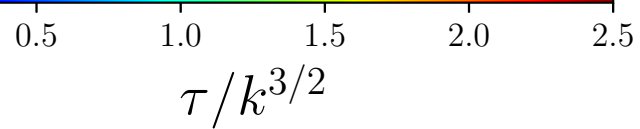

FIG. 5. As Fig. 1, for the SGR model. Initial conditions are as in Fig. 2, and other simulation parameters are $N_{q} \times N_{p}=700 \times 1024$, $\Delta t=2.5 \times 10^{-3}$, and $t_{\max }=1.5 \times 10^{3}$.

freedom and Hamiltonian $H\left(q_{1}, \ldots, q_{d}, p_{1}, \ldots, p_{d}\right)$. Being, by definition, that

$$
\begin{aligned}
\{f, g\} & =\sum_{j=1}^{d}\left(\frac{\partial f}{\partial p_{j}} \frac{\partial g}{\partial q_{j}}-\frac{\partial f}{\partial q_{j}} \frac{\partial g}{\partial p_{j}}\right) \\
& =\sum_{j=1}^{d} \frac{\partial}{\partial q_{j}}\left(\frac{\partial f}{\partial p_{j}} g\right)+\sum_{j=1}^{d} \frac{\partial}{\partial p_{j}}\left(-\frac{\partial f}{\partial q_{j}} g\right),
\end{aligned}
$$

which is a divergence in phase space, we have

$$
\int\{f, g\} d \Gamma=0
$$

for any $f$ and $g$ decaying sufficiently rapidly for large values of coordinates and momenta. In Eq. (A2) the integral is extended to the whole $2 d$-dimensional phase space and we have used the shorthand notation $d \Gamma=d p d q=$ $\prod_{i=1}^{d} d p_{i} d q_{i}$, which we continue to use henceforth. From Eq. (A2), considering three functions, $f, g$, and $h$, again decaying sufficiently rapidly at infinity, and applying the Leibnitz rule,

$$
\{f, g h\}=g\{f, h\}+h\{f, g\},
$$

we get the integration-by-parts formula

$$
\int h\{f, g\} d \Gamma=-\int g\{f, h\} d \Gamma .
$$

\section{Conservation laws in coarse-grained evolution}

\section{a. Conservation of the norm of $f$}

Using the identity

$$
\{M(H), \cdot\}=M^{\prime}(H)\{H, \cdot\},
$$

Eq. (7) becomes

$$
\frac{\partial \tilde{f}}{\partial t}=\{H, \tilde{f}\}+\sum_{n=2}^{\infty}\left\{M_{n},\{H, \cdot\}^{n-1}\right\} \tilde{f},
$$

where the $M_{n}$ 's are such that $M_{n}^{\prime}(H)=\mu_{n}(H)$. Integrating over the whole phase space and using Eq. (A2) we have

$$
\int \frac{\partial \tilde{f}}{\partial t} d \Gamma=0
$$

and being that $\int \partial_{t} \tilde{f} d \Gamma=\frac{d}{d t} \int \tilde{f} d \Gamma$, Eq. (A7) implies the conservation of the norm.

\section{b. Conservation of the energy}

Working in one dimension to ease the notation, the energy of the system in a state defined by the coarse-grained distribution can be written as

$$
\begin{aligned}
E[\tilde{f}]= & \frac{1}{2} \int p^{2} \tilde{f}(p, q) d \Gamma \\
& +\frac{1}{2} \iint \tilde{f}(p, q) V\left(q-q^{\prime}\right) \tilde{f}\left(p^{\prime}, q^{\prime}\right) d \Gamma d \Gamma^{\prime},
\end{aligned}
$$


leading to

$$
\begin{aligned}
\frac{\delta E}{\delta \tilde{f}} & =\frac{p^{2}}{2}+\int V\left(q-q^{\prime}\right) \tilde{f}\left(q^{\prime}, p^{\prime}\right) d \Gamma^{\prime} \\
& =\frac{p^{2}}{2}+\tilde{U}(q) \equiv H[\tilde{f}] .
\end{aligned}
$$

The time derivative of the energy is

$$
\frac{d}{d t} E[\tilde{f}]=\int \frac{\delta E}{\delta \tilde{f}} \frac{\partial \tilde{f}}{\partial t} d \Gamma,
$$

so that Eq. (A9) implies

$$
\frac{d}{d t} E[\tilde{f}]=\int H[\tilde{f}] \frac{\partial \tilde{f}}{\partial t} d \Gamma .
$$

Using Eq. (A6) we have

$$
H \frac{\partial \tilde{f}}{\partial t}=\frac{1}{2}\left\{H^{2}, \tilde{f}\right\}+\sum_{n=2}^{\infty}\left\{\mathcal{M}_{n},\{H, \cdot\}^{n-1}\right\} \tilde{f},
$$

where the $\mathcal{M}_{n}(H)$ are such that $\mathcal{M}_{n}^{\prime}(H)=H \mu_{n}(H)$; integrating the above equation over the whole phase space and using Eq. (A11) we get $d E / d t=0$.

\section{Time evolution of convex Casimirs}

The fine-grained Vlasov evolution has infinite conserved quantities (Casimirs) obtained by integrating a generic function $C(f)$ of the distribution function $f$ over the whole phase space. Replacing $f$ with a coarse-grained one the Casimirs are no longer constant in motion. However, among all the Casimirs defined using any coarse-graining distribution function $\tilde{f}$, that is,

$$
\mathcal{C}[\tilde{f}]=\int C(\tilde{f}) d \Gamma,
$$

those corresponding to a convex $C$ (which are referred to as "convex Casimirs" henceforth) must be nonincreasing functions of time [24]. In the case of one-dimensional systems, considered below, we are able to prove that our version of the coarse-grained dynamics does agree with such a constraint (see Sec. A 2 b below). We did not succeed in proving this result for the most general form, (7), of the coarse-grained evolution equation, but we can show that convex Casimirs do not increase with time if we restrict ourselves to the lowestorder truncation of Eq. (7).

\section{a. General case}

Let us consider the lowest-order truncation of Eq. (7), that is,

$$
\frac{\partial \tilde{f}}{\partial t}=\{H, \tilde{f}\}+\mu_{2}(H)\{H,\{H, \tilde{f}\}\},
$$

provided that $\mu_{2}(H) \geqslant 0$. Indeed,

$$
\begin{aligned}
\frac{d}{d t} \mathcal{C}[\tilde{f}]= & \int C^{\prime}(\tilde{f}) \frac{\partial \tilde{f}}{\partial t} d \Gamma=\int C^{\prime}(\tilde{f})\{H, \tilde{f}\} d \Gamma \\
& +\int \mu_{2}(H) C^{\prime}(\tilde{f})\{H,\{H, \tilde{f}\}\} d \Gamma,
\end{aligned}
$$

and using Eq. (A5), with $\mu_{2}(H)=M_{2}^{\prime}(H)$, we get

$$
\frac{d}{d t} \mathcal{C}[\tilde{f}]=\int\{H, C(\tilde{f})\} d \Gamma+\int C^{\prime}(\tilde{f})\left\{M_{2}(H),\{H, \tilde{f}\}\right\} d \Gamma .
$$

The first integral on the r.h.s. of the above equation vanishes due to Eq. (A2), while integrating by parts the second term using Eq. (A4) we get

$$
\begin{aligned}
\frac{d}{d t} \mathcal{C}[\tilde{f}] & =-\int\left\{M_{2}(H), C^{\prime}(\tilde{f})\right\}\{H, \tilde{f}\} d \Gamma \\
& =-\int \mu_{2}(H) C^{\prime \prime}(\tilde{f})\{H, \tilde{f}\}^{2} d \Gamma ;
\end{aligned}
$$

$C(\tilde{f})$ being convex, this implies $\dot{\mathcal{C}}[\tilde{f}] \leqslant 0$ provided that $\mu_{2}(H) \geqslant 0$. It is interesting to note that Eq. (A17) tells us that $\mathcal{C}[\tilde{f}]$ does not reach its minimum: its evolution eventually stops when $\tilde{f}$ approaches a stationary solution, that is, such that $\{H, \tilde{f}\}=0$. The latter is a necessary feature of a consistent evolution, because it would not be possible, in general, to reach a state where all the (infinite) convex Casimirs are simultaneously minimized (see also the discussion in Ref. [24]).

\section{b. One-dimensional systems}

Let us now show that the evolution defined by Eqs. (13) and (14) fulfills the constraint on the evolution of convex Casimirs. To this end we explicitly write down the average in Eq. (14) in terms of action-angle variables at time $t$,

$$
\begin{aligned}
\left\langle e^{\Delta t\{H,\}}\right\rangle_{\Delta \Gamma} & =\frac{1}{\Delta J \Delta \vartheta} \int_{\Delta J} d J^{\prime} \int_{\Delta \vartheta} d \vartheta^{\prime} e^{-\omega\left(J^{\prime}\right) \Delta t \partial_{\vartheta}} \\
& =\frac{1}{\Delta J} \int_{\Delta J} d J^{\prime} e^{-\omega\left(J^{\prime}\right) \Delta t \partial_{\vartheta}},
\end{aligned}
$$

where we have dropped the average over the angle variable since the integrand depends only on $J^{\prime}$. Being $e^{-\Delta t \omega(J) \partial_{\vartheta}}$ the translation operator along the direction $\vartheta$ the time-evolved $\tilde{f}_{t+\Delta t}$ can be written in terms of $\tilde{f}_{t}$ as

$$
\tilde{f}_{t+\Delta t}(\vartheta, J)=\frac{1}{\Delta J} \int_{\Delta J} d J^{\prime} \tilde{f}(\vartheta-\Delta t \omega(J), J) .
$$

which in turn can be expressed as an average on the random variabile $J$,

$$
\tilde{f}_{t+\Delta t}(\vartheta, J)=\left\langle\tilde{f}\left(\vartheta-\omega\left(J^{\prime}\right) \Delta t, J\right)\right\rangle_{\Delta J} .
$$

On the other hand, for any convex function $C(x)$ and for any random variable $x$,

$$
C(\langle x\rangle) \leqslant\langle C(x)\rangle,
$$

so that

$$
C\left(\tilde{f}_{t+\Delta t}\right) \leqslant\langle C(\tilde{f}(\vartheta-\omega(J) \Delta t, J))\rangle_{\Delta J}
$$

or, explicitly writing the average over $\Delta J$ once again,

$$
C\left(\tilde{f}_{t+\Delta t}\right) \leqslant \frac{1}{\Delta J} \int_{\Delta J} d J^{\prime} C\left(\tilde{f}\left(\vartheta-\omega\left(J^{\prime}\right) \Delta t, J\right)\right) .
$$

To obtain a condition on the Casimir functional at time $t+\Delta t$ we have to integrate the above relation in $\vartheta$ and $J$ all over the 

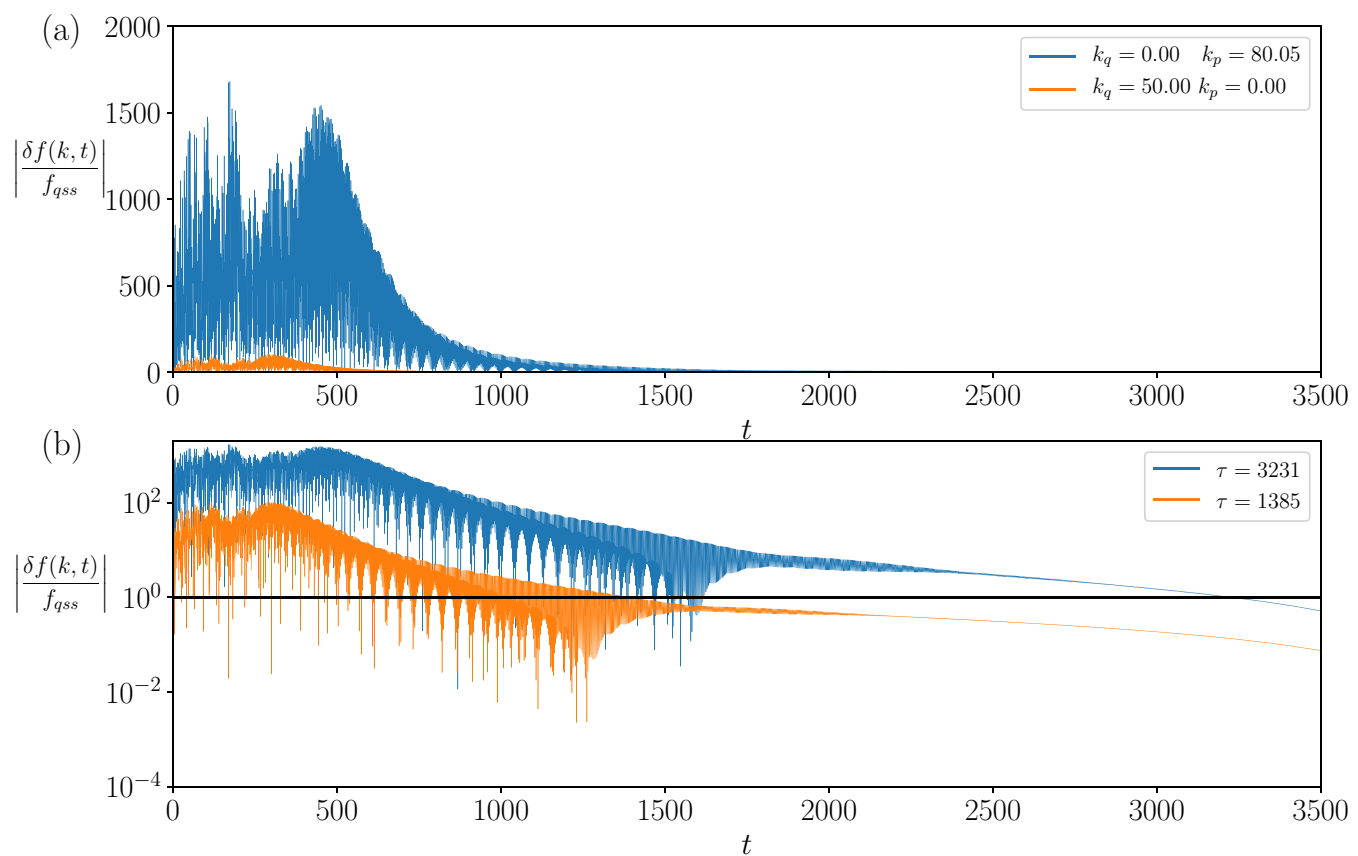

FIG. 6. HMF model. (a) Examples of $\left|\left[f(\mathbf{k}, t)-f^{\mathrm{qss}}(\mathbf{k})\right] / f^{\mathrm{qss}}(\mathbf{k})\right|$ (see legend for the values of $\mathbf{k}$ ), on a linear scale. (b) As (a), but on a log-linear scale. The horizontal black line defines our threshold (equal to 1 here); the damping time $\tau$ is such that the curve is below the horizontal line for any $t>\tau$, and the values of $\tau$ obtained for the two cases plotted are reported in the legend. Simulation parameters are as in Fig. 1, i.e., $N_{q} \times N_{p}=1064 \times 1248, \Delta t=2.5 \times 10^{-3}$, and $t_{\max }=4 \times 10^{3}$.

phase space, obtaining

$\mathcal{C}\left[\tilde{f}_{t+\Delta \tau}\right] \leqslant \frac{1}{\Delta J} \int_{\Delta J} d J^{\prime} \int d J \int d \vartheta C\left(\tilde{f}\left(\vartheta-\omega\left(J^{\prime}\right) \Delta t, J\right)\right)$,

but $\tilde{f}$ is a periodic function of $\vartheta$, so that

$$
\int d \vartheta C\left(\tilde{f}\left(\vartheta-\omega\left(J^{\prime}\right) \Delta t, J\right)\right)=\int d \vartheta C(\tilde{f}(\vartheta, J)),
$$

which no longer depends on $J^{\prime}$; the average over $\Delta J$ is thus trivial and Eq. (A24) becomes

$$
\mathcal{C}\left[\tilde{f}_{t+\Delta \tau}\right] \leqslant \mathcal{C}\left[\tilde{f}_{t}\right],
$$

that is, what we wanted to prove.

\section{APPENDIX B: LANGEVIN EQUATION}

It is interesting to note that the leading-order effective evolution equation in the one-dimensional case, (26), can be cast in the form of a Fokker-Planck equation and interpreted, in turn, in the corresponding Langevin formalism. The nested Poisson bracket in Eq. (26) can be written as

$$
\{\omega(H),\{\omega(H), \tilde{f}\}\}=\partial_{x_{i}}\left(v_{i} \partial_{x_{j}}\left(v_{j} f\right)\right),
$$

where $i=1,2, x_{1}=q, x_{2}=p$, and $v_{i}=\varepsilon_{i j} \partial_{x_{i}} \omega$, with $\varepsilon_{i j}$ the totally antisymmetric Levi-Civita symbol, and we have used the Einstein summation convention over repeated indices, so that Eq. (26) becomes

$$
\frac{\partial \tilde{f}}{\partial t}=-\varepsilon_{i j} \partial_{x_{i}}\left(\partial_{x_{j}} H \tilde{f}\right)+\frac{1}{24} \Delta t(\Delta J)^{2} \partial_{x_{i}}\left(v_{i} \partial_{x_{j}}\left(v_{j} \tilde{f}\right)\right),
$$

in which we recognize the general form of a Fokker-Planck equation with a nonisotropic and nonuniform diffusion coef- ficient. This equation is in turn equivalent, in the Langevin formalism, to the Stratonovich differential equation [42]

$$
\dot{x}_{i}=\varepsilon_{i j} \partial_{x_{j}} H+v_{j} \xi(t),
$$

$\xi(t)$ being a white noise with the correlation function

$$
\left\langle\xi(t) \xi\left(t^{\prime}\right)\right\rangle=\frac{1}{24} \Delta J^{2} \Delta t \delta\left(t-t^{\prime}\right) .
$$

Exploiting the definition of $v_{j}$ we can write

$$
\begin{aligned}
& \dot{q}=\frac{\partial H}{\partial p}+\frac{\partial \omega(H)}{\partial p} \xi(t), \\
& \dot{p}=-\frac{\partial H}{\partial q}-\frac{\partial \omega(H)}{\partial q} \xi(t) ;
\end{aligned}
$$

as expected, this pair of equations can be derived from the stochastic Hamiltonian

$$
\tilde{H}=H+\omega(H) \xi(t),
$$

where once again we are using the Stratonovich formalism.

\section{APPENDIX C: MEASURING DAMPING TIMES IN NUMERICAL SIMULATIONS}

We have defined the damping time $\tau_{\mathbf{k}}$ as the time for which the deviation of $f(\mathbf{k}, t)$ from its asymptotic value $f^{\mathrm{qss}}(\mathbf{k})$ is definitively smaller than $f^{\text {qss }}(\mathbf{k})$ itself, that is, $\tau_{\mathbf{k}}$ is such that

$$
\left|\frac{\delta f(\mathbf{k}, t)}{f^{\mathrm{qss}}(\mathbf{k})}\right| \equiv\left|\frac{f(\mathbf{k}, t)-f^{\mathrm{qss}}(\mathbf{k})}{f^{\mathrm{qss}}(\mathbf{k})}\right|<1, \quad \forall t \geqslant \tau_{\mathbf{k}} .
$$

The asymptotic value $f^{\mathrm{qss}}(\mathbf{k})$ is defined as the average of $f(\mathbf{k}, t)$ over the final part of the simulation, of duration $t_{0}=$ 250. In Fig. 6 we report the time evolution of $[f(\mathbf{k}, t)-$ 
(a)

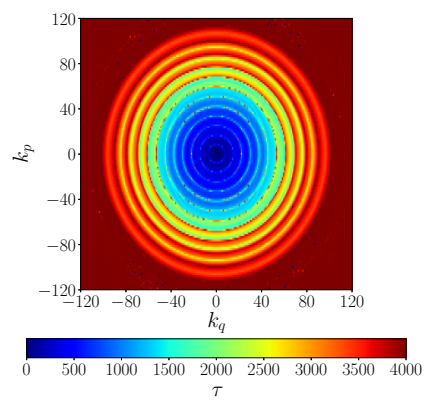

(c)

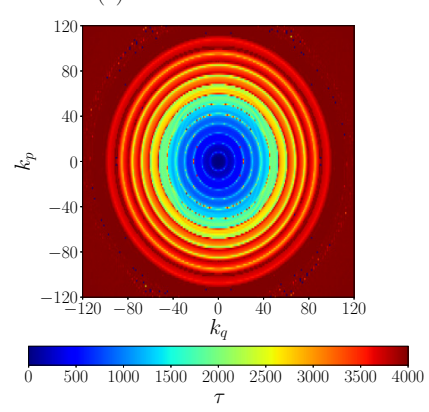

(b)

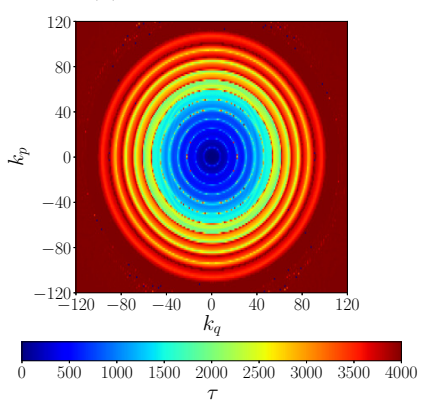

(d)

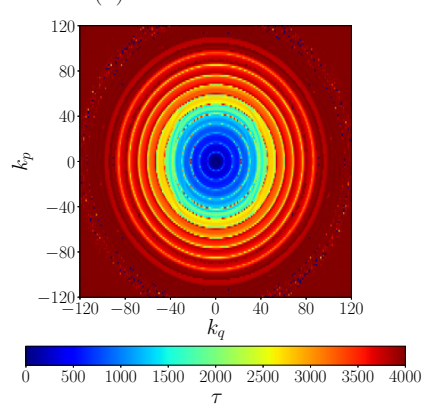

(e)

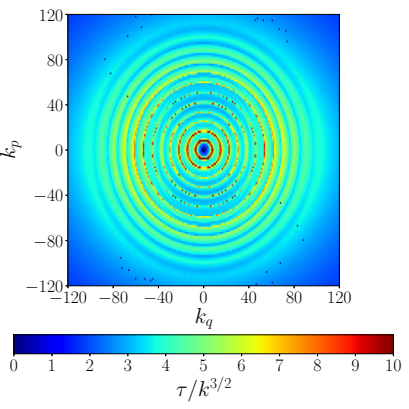

(g)

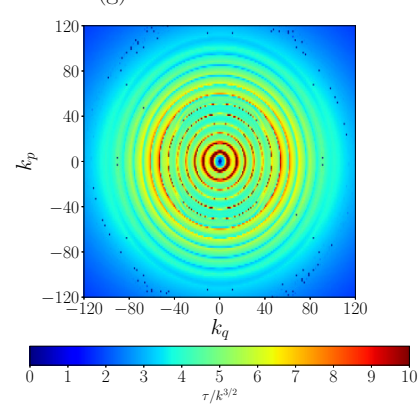

(f)

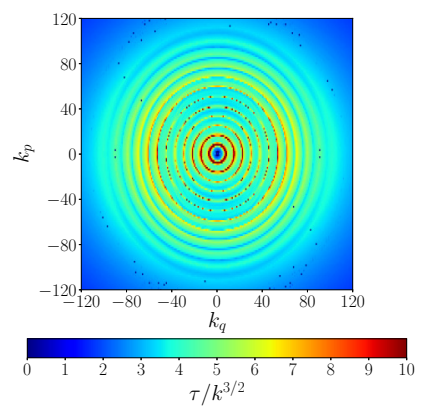

(h)

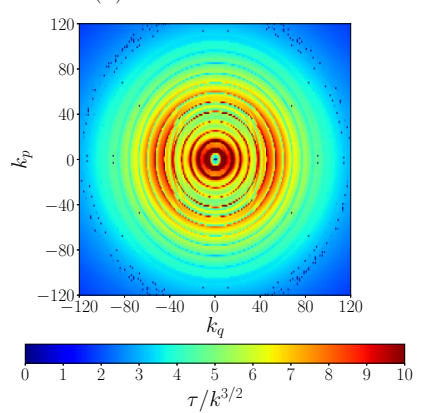

FIG. 7. Dependence of computed and rescaled damping times on the threshold used to define the damping time [see Eq. (C1)] for the HMF model. We show here how the results reported in Fig. 1 are affected by the choice of different thresholds. (a-d) Plots of the damping times $\tau$ as a function of $\mathbf{k}$ for four values of the threshold: 1.0 (a), $0.75(\mathrm{~b}), 0.50$ (c), and $0.25(\mathrm{~d})$. (e-h) Plots of the corresponding rescaled damping times $\tau(\mathbf{k}) / k^{3 / 2}$ : values of the threshold are $1.0(\mathrm{e}), 0.75(\mathrm{f}), 0.50(\mathrm{~g})$, and $0.25(\mathrm{~h})$. Note the change of scale between (a)-(d) and (e)-(h). Simulation parameters as in Fig. 6.

$\left.f^{\mathrm{qss}}(\mathbf{k})\right] / f^{\mathrm{qss}}(\mathbf{k})$ for two particular Fourier components (extracted from the simulation used to obtain the results shown in Fig. 1) to clarify the definition of the damping times. The threshold we used, that is, the fact that the r.h.s. of the inequality in Eq. (C1) equals 1, is somewhat arbitrary, and any other number not as far from unity would make sense. For this reason we show in Fig. 7 how the results presented in
Fig. 1 are affected by the choice of different threshold values. It is apparent that a smaller threshold implies longer damping times, but the damping times still follow the scaling $\tau_{\mathbf{k}} \propto$ $k^{3 / 2}$ with more or less the same accuracy (perhaps getting only slightly worse for smaller thresholds) for any choice of threshold. In all the results presented in this paper the threshold has been kept equal to 1 as in Eq. (C1).
[1] A. Campa, T. Dauxois, D. Fanelli, and S. Ruffo, Physics of Long-Range Interacting Systems (Oxford University Press, Oxford, UK, 2014).

[2] A. Campa, T. Dauxois, and S. Ruffo, Phys. Rep. 480, 57 (2009).

[3] J. Binney and S. Tremaine, Galactic Dynamics, 2nd ed. (Princeton University Press, Princeton, NJ, 2008).

[4] D. R. Nicholson, Introduction to Plasma Theory (Wiley, New York, 1983).

[5] S. Schütz and G. Morigi, Phys. Rev. Lett. 113, 203002 (2014).

[6] S. Gupta and L. Casetti, New J. Phys. 18, 103051 (2016).

[7] F. Bouchet and A. Venaille, Phys. Rep. 515, 227 (2012).

[8] Y. Levin, R. Pakter, F. B. Rizzato, T. N. Teles, and F. P. C. Benetti, Phys. Rep. 535, 1 (2014).

[9] I. Latella, A. Pérez-Madrid, A. Campa, L. Casetti, and S. Ruffo, Phys. Rev. Lett. 114, 230601 (2015).

[10] I. S. Chandrasekhar, Astrophys. J. 93, 285 (1941).

[11] P. J. Morrison, Phys. Lett. A 80, 383 (1980).

[12] J. Perez and J.-J. Aly, Mon. Not. Royal Astron. Soc. 280, 689 (1996).

[13] H. E. Kandrup, Astrophys. J. 500, 120 (1998).
[14] M. Hénon, Ann. Astrophysique 27, 83 (1964).

[15] T. S. van Albada, Mon. Not. R. Astron. Soc. 201, 939 (1982).

[16] F. Sylos Labini, Mon. Not. R. Astron. Soc. 423, 1610 (2012).

[17] D. Lynden-Bell, Mon. Not. R. Astron. Soc. 136, 101 (1967).

[18] L. Casetti and S. Gupta, Eur. Phys. J. B 87, 91 (2014).

[19] T. N. Teles, S. Gupta, P. Di Cintio, and L. Casetti, Phys. Rev. E 92, 020101(R) (2015).

[20] P. Di Cintio, S. Gupta, and L. Casetti, Mon. Not. R. Astron. Soc. 475, 1137 (2018).

[21] G. Giachetti and L. Casetti, J. Stat. Mech.: Theory Exp. (2019) 043201.

[22] J. Barré, A. Olivetti, and Y. Y. Yamaguchi, J. Stat. Mech.: Theory Exp. (2010) P08002.

[23] J. Barré, A. Olivetti, and Y. Y. Yamaguchi, J. Phys. A: Math. Theor. 44, 405502 (2011).

[24] S. Tremaine, M. Hénon, and D. Lynden-Bell, Mon. Not. R. Astron. Soc. 219, 285 (1986).

[25] P. de Buyl, Comput. Phys. Commun. 185, 1822 (2014). 
[26] E. Sonnendrücker, J. Roche, P. Bertrand, and A. Ghizzo, J. Comput. Phys. 149, 201 (1999).

[27] M. Antoni and S. Ruffo, Phys. Rev. E 52, 2361 (1995).

[28] Y. Sota, O. Iguchi, M. Morikawa, T. Tatekawa, and K.-i. Maeda, Phys. Rev. E 64, 056133 (2001).

[29] T. Tatekawa, F. Bouchet, T. Dauxois, and S. Ruffo, Phys. Rev. E 71, 056111 (2005).

[30] C. Nardini and L. Casetti, Phys. Rev. E 80, 060103(R) (2009).

[31] L. Casetti and C. Nardini, J. Stat. Mech.: Theory Exp. (2010) P05006.

[32] B. Monechi and L. Casetti, Phys. Rev. E 86, 041136 (2012).

[33] J. L. Tennyson, J. D. Meiss, and P. J. Morrison, Physica D: Nonlin. Phenom. 71, 1 (1994).

[34] R. Bonifacio, F. Casagrande, G. Cerchioni, L. Salvo Souza, P. Pierini, and N. Piovella, Riv. Nuovo Cimento 13, 1 (1990).
[35] S. D. Mathur, Mon. Not. R. Astron. Soc. 243, 529 (1990).

[36] M. D. Weinberg, Astrophys. J. 373, 391 (1991).

[37] X. Leoncini, T. L. Van Den Berg, and D. Fanelli, Europhys. Lett. 86, 20002 (2009).

[38] R. Robert and J. Sommeria, Phys. Rev. Lett. 69, 2776 (1992).

[39] P.-H. Chavanis and J. Sommeria, Phys. Rev. Lett. 78, 3302 (1997).

[40] P.-H. Chavanis and F. Bouchet, Astron. Astrophys. 430, 771 (2005).

[41] L. Beraldo e Silva, W. de Siqueira Pedra, and M. Valluri, Astrophys. J. 872, 20 (2019).

[42] N. G. van Kampen, Stochastic Processes in Physics and Chemistry (North-Holland, New York, 2007). 